\title{
Cross-Modal Reorganization of Horizontal Connectivity in Auditory Cortex without Altering Thalamocortical Projections
}

\author{
W.-J. Gao and S. L. Pallas \\ Department of Biology, Georgia State University, Atlanta, Georgia 30302
}

The development of the different, highly specialized regions of the mammalian cerebral cortex depends in part on neural activity, either intrinsic spontaneous activity or externally driven sensory activity. To determine whether patterned sensory activity instructs the development of intrinsic cortical circuitry, we have experimentally altered the modality of sensory inputs to cerebral cortex. Neonatal diversion of retinal axons to the auditory thalamus (cross-modal rewiring) results in a primary auditory cortex (Al) that resembles visual cortex in its response properties and topography (Roe et al., 1990, 1992). To test the hypothesis that the visual response properties are created by a visually driven reorganization of auditory cortical circuitry, we investigated the effect of early visual experience on the development of intrinsic, horizontal connections within Al. Horizontal connections are likely to play an important role in the construc- tion of visual response properties in Al as they do in visual cortex. Here we show that early visual inputs to auditory thalamus can reorganize horizontal connections in Al, causing both an increase in their extent and a change in pattern, so that projections are not restricted to the isofrequency axis, but extend in a more isotropic pattern around the injection site. Thus, changing afferent modality, without altering the source of the thalamocortical axons, can profoundly alter cortical circuitry. Similar changes may underlie cortical compensatory processes in deaf or blind humans and may also have played a role in the parcellation of neocortex during mammalian evolution.

Key words: cortical development; sensory cortex; crossmodal plasticity; retinal axons; activity; biotinylated dextran amine; visual development; cortical circuitry; ferret
The role of sensory inputs in specifying the regional characteristics of cerebral cortex has been a matter of considerable debate among developmental neuroscientists (Rakic, 1988; O'Leary, 1989; Pallas, 1990; Sur et al., 1990; Levitt et al., 1997). The identity and pattern of activity within the thalamocortical inputs may play an important role in cortical regionalization (Katz and Shatz, 1996; Catalano and Shatz, 1998). To test the hypothesis that certain aspects of cortical regional identity are instructed by thalamic activity patterns, independent of molecular cues on thalamocortical axons, we investigated whether the intrinsic circuitry of sensory cortex can be altered by changing the modality but not the source of thalamocortical inputs during development. By diverting the retina to the auditory thalamus (MGN) early in development, the modality and thus activity pattern of the inputs to auditory cortex can be altered without manipulating the thalamocortical pathway that carries the novel information. This "cross-modal rewiring" paradigm also allows us to ask whether different cortical areas are functionally interchangeable if they are driven by the same sensory epithelium. Previous results have shown that auditory cortex that has received visual input during postnatal development resembles primary visual cortex in its topography and receptive field properties (Sur et al., 1988; Roe et al., 1990, 1992). Our goal was to determine whether this similarity

\footnotetext{
Received Feb. 5, 1999; revised June 18, 1999; accepted June 30, 1999.
}

This work was supported by grants to S.L.P. from the National Science Foundation, the Whitehall Foundation, the Fight for Sight Inc. Research Division of Prevent Blindness America, and the Georgia Research Alliance. We thank Astou Coly and Karen Page for their technical expertise, and Reha Erzurumlu, Barbara Finlay, Paul Katz, and Joanne Matsubara for their helpful criticisms of this manuscript.

Correspondence should be addressed to S. L. Pallas, Department of Biology, Georgia State University, P.O. Box 4010, Atlanta, GA 30302.

Dr. Gao's present address: Section of Neurobiology, Yale University School of Medicine, 333 Cedar Street, New Haven, CT 06510.

Copyright @ 1999 Society for Neuroscience 0270-6474/99/197940-11\$05.00/0 is caused by a modification of the intrinsic circuitry of the AI by the visual inputs during development, or alternatively, whether the visual afferents make use of pre-existing similarities in processing circuitry between visual and auditory cortex.

Each sensory cortical area has a number of unique structural and functional attributes. The pattern of horizontal connections between neurons within a cortical area is one striking example. These connections may be important in constructing the characteristic receptive field properties seen in each region of sensory cortex. In mammalian visual cortex, horizontal projections are arranged in a roughly circular pattern, depending on the species (Rockland and Lund, 1982; Gilbert and Wiesel, 1983; Matsubara et al., 1985, 1987; Callaway and Katz, 1990; Weliky and Katz, 1994; Durack and Katz, 1996; Ruthazer and Stryker, 1996; Bosking et al., 1997), and connect neurons with similar orientation tuning (Gilbert and Wiesel, 1979; T'so et al., 1986; Gilbert and Wiesel, 1989; Malach et al., 1993). In auditory cortex, neurons within an isofrequency domain are interconnected, forming an elongated strip of horizontally projecting axons (Reale et al., 1983; Imig et al., 1986; Matsubara and Phillips, 1988; Ojima et al., 1991; Wallace and Bajwa, 1991). Early in development, horizontal projections are widespread, but become progressively more restricted under the influence of neural activity (Callaway and Katz, 1990, 1991; Weliky and Katz, 1994; Ruthazer and Stryker, 1996). This study addresses whether the organization of specific horizontal connections can be altered by changing the modality and thus the patterned activity of thalamocortical inputs during development. Alterations in horizontal connections may play an important role in organizing the visual receptive field properties and retinotopy that have been reported in cross-modal AI (Roe et al., 1990, 1992). 


\section{MATERIALS AND METHODS}

The effect of early visual inputs on the pattern of horizontal connectivity in auditory cortex was investigated by making small injections of biotinylated dextran amine (BDA) in AI of adult cross-modal and normal ferrets. Specific procedures are detailed below.

Animals. Timed pregnant ferrets were purchased from Marshall Farms (North Rose, NY), and their kits were used in the cross-modal group. Normal animals were purchased as adults. All animals were kept on a 16/8 light/dark cycle and fed ferret chow and water ad libitum. The animals were treated in accordance with all institutional (Institutional Animal Care and Use Committee), Society for Neuroscience, and $\mathrm{Na}-$ tional Institutes of Health guidelines for Animal Care and Laboratory Use. Fifteen control and twelve experimental ferrets were used in this study. Seven representative cases from each group were analyzed quantitatively. Excluded cases had poor injections, insufficient transport, or small lesions.

Neonatal induction of cross-modal projections. To route retinal axons to the auditory thalamus and thus alter the modality of thalamocortical input, specific lesions were made in ferret kits (Sur et al., 1988; Angelucci et al., 1998). The aim was to reduce normal targets of the retina and to deafferent the medial geniculate nucleus (MGN; auditory thalamus), thus inducing the retinal axons to innervate MGN and provide the auditory pathway with visual information. Within $24 \mathrm{hr}$ after birth, neonates were placed on a heating pad and anesthetized with isoflurane. A small incision was made along the midline of the scalp, and the midbrain was exposed by removing a flap of bone. A heat cautery was used to ablate the inferior colliculus (IC) bilaterally, the superficial part of the left superior colliculus (SC), and the left visual cortex (V1). In addition, the brachium of the inferior colliculus (BIC) was severed. After recovery from the surgery, the kits were returned to the animal colony. Supportive care and analgesics were given as necessary throughout the recovery period.

$B D A$ tracer injections. Biotinylated dextran amine was chosen as a tracer to reveal horizontal projections because of its ease of use, its ability to travel anterogradely, and its stability. After administration of atropine $(0.04 \mathrm{mg} / \mathrm{kg})$ and doxapram $(2 \mathrm{mg} / \mathrm{kg})$, adult ferrets $(120 \mathrm{~d}$ or older) were preanesthetized with ketamine $(40 \mathrm{mg} / \mathrm{kg})$ combined with xylazine $(1-2 \mathrm{mg} / \mathrm{kg})$ or diazepam $(2 \mathrm{mg} / \mathrm{kg})$. Amoxicillin $(30 \mathrm{mg} / \mathrm{kg})$ was then injected as a prophylactic against infection, followed by dexamethasone $(40 \mathrm{mg} / \mathrm{kg})$ to prevent brain edema. An endotracheal tube was placed to deliver isoflurane anesthesia and oxygen, and the cephalic vein was cannulated for fluid therapy during the surgery. The head was fixed in a stereotaxic device, and electrodes were placed for monitoring of heart rate and respiration. A deep state of anesthesia was maintained with $1.25-2 \%$ isoflurane. The surgery was then performed under strict aseptic conditions. A flap of skin and muscle was removed from above the lateral cortex. A hole was drilled in the skull to expose the primary auditory cortex (AI), and the dura was retracted. A volume of 50-150 nl of $10 \% \mathrm{BDA}$ in sterile saline was injected through a micropipette (tip diameter, 15-25 $\mu \mathrm{m}$ ) with the aid of a Picospritzer II (General Valve, Fairfield, NJ). One injection per hemisphere was made 300-500 $\mu \mathrm{m}$ below the pia, into the superficial layer $2 / 3$ of AI, approximately in the center of the ectosylvian gyrus where $\mathrm{AI}$ is located (Fig. $1 A$ ) (Kelly et al., 1986). The BDA was injected slowly over $15-20 \mathrm{~min}$. The opening in the skull was then covered by a piece of sterile plastic film attached with dental cement. The muscle was sutured, and the incision was closed. The ferret was supervised closely until it recovered from the anesthesia, and was given supportive care and analgesics as necessary throughout the recovery period.

Tracing of retinal projections. To ensure that the visual inputs were successfully routed into the MGN in cross-modal animals, cholera toxin (B subunit, 1\%, $6 \mu \mathrm{l}$; List Biological Labs, Campbell, CA) was injected into the posterior chamber of each eye at the end of the BDA injection surgery. Several normal animals were also given eye injections as a control (Pallas and Moore, 1997). Animals were monitored postsurgically and given topical ophthalmic antibiotics and anti-inflammatory drugs (dexamethasone, $40 \mathrm{mg} / \mathrm{kg}$ ) if necessary.

Histology and immunocytochemistry. After 4-5 d survival, animals were overdosed with sodium pentobarbital $(65 \mathrm{mg} / \mathrm{kg})$ and perfused through the heart with $0.1 \mathrm{M}$ PBS followed by $2 \%$ paraformaldehyde for $10 \mathrm{~min}$. The brains were quickly extracted from the skull, and the thalamus and midbrain were separated from each hemisphere. The cortical hemispheres were then gently flattened between two weighted glass plates. After flattening, the tissues were post-fixed with $4 \%$ paraformaldehyde in $30 \%$ sucrose $/ 0.1 \mathrm{M}$ phosphate buffer $(\mathrm{PB})$ at $4^{\circ} \mathrm{C}$ for $24 \mathrm{hr}$. The brain tissue was then sectioned frozen at $50 \mu \mathrm{m}$ in a
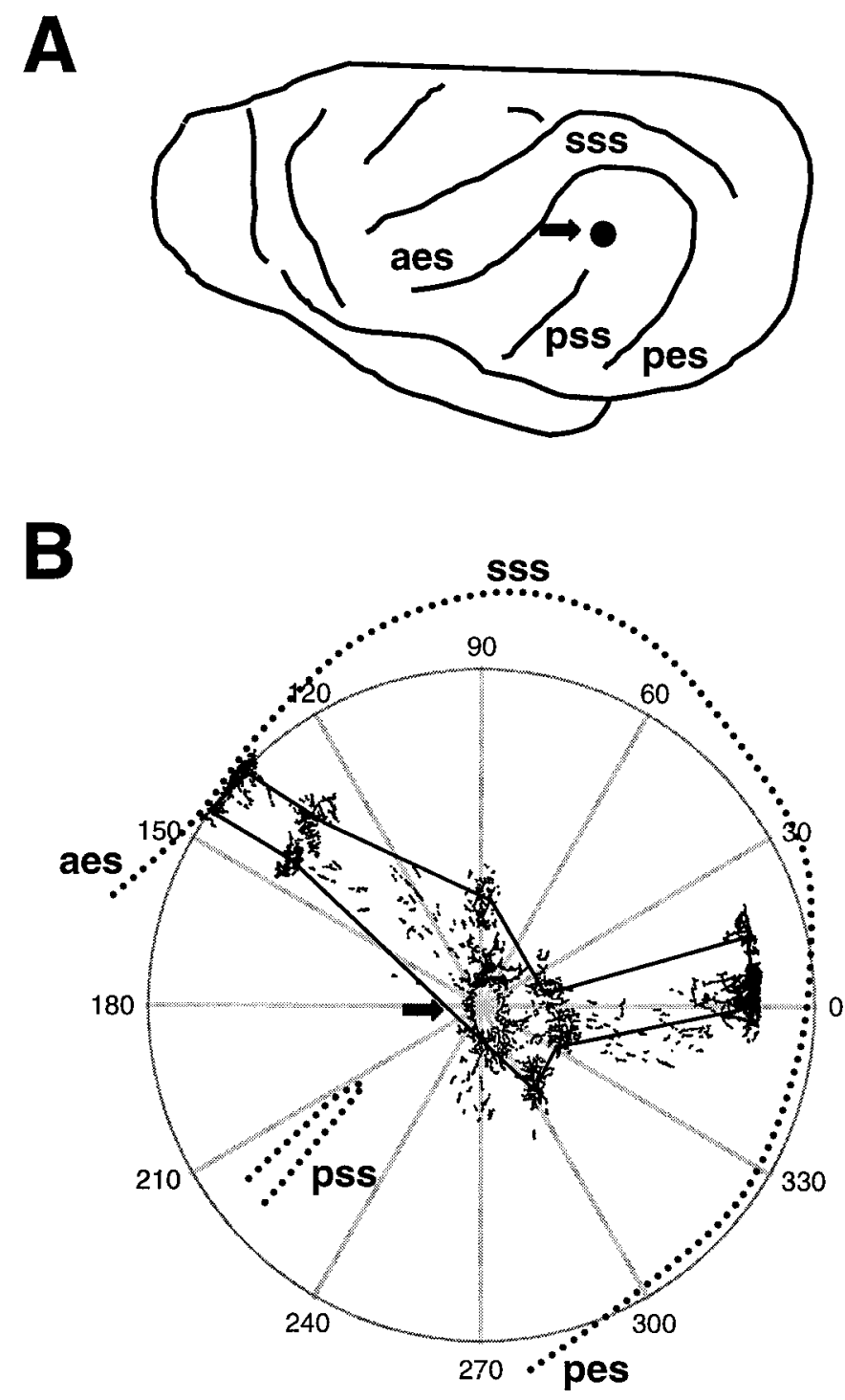

Figure 1. A, Drawing of the left side of a ferret brain, with the location of the sulci surrounding AI noted. The arrow shows the approximate location of the tracer injections. aes, Anterior ectosylvian sulcus; pes, posterior ectosylvian sulcus; sss, suprasylvian sulcus; $p s s$, pseudosylvian sulcus. $B$, Illustration of methods used for making polar plots of bouton distribution. The arrow shows the tracer injection site, and the camera lucida drawing of the resulting label is superimposed on the polar plot. With the injection site as the origin, the polar coordinates of the center of each bouton cluster were recorded. Lines were drawn through the center of each bouton cluster to arrive at the plots shown in Figures 6 and 7. The dashed lines indicate the sulci surrounding AI and show how the plots were oriented.

tangential (cortical hemispheres) or coronal (midbrain and thalamus) plane. The sections were collected in $0.1 \mathrm{M} \mathrm{PB}$. One set of sections at $200 \mu \mathrm{m}$ intervals was mounted onto gelatin-subbed slides for Nissl staining with cresylecht violet. The other sections were used for immunocytochemistry.

To visualize the anterograde labeling resulting from the BDA injections, the cortical sections were rinsed with $0.1 \mathrm{M}$ PBS plus $0.3 \%$ Triton $\mathrm{X}-100$ three times for $15 \mathrm{~min}$, then left in same solution for $1 \mathrm{hr}$. They were then reacted with $\mathrm{ABC}$ complex (Vectastain Elite; Vector Laboratories, Burlingame, CA) for $1-1.5 \mathrm{hr}$ at a dilution of 1:500 in $0.1 \mathrm{M}$ PBS containing $0.3 \%$ Triton $\mathrm{X}-100$. After thoroughly rinsing in buffer, a diaminobenzidine (DAB) reaction with $0.02 \%$ DAB and $0.008 \%$ hydrogen peroxide was performed. The reactions were intensified by adding $2 \%$ nickel ammonium sulfate and $0.68 \%$ imidazole (Tago et al., 
1989). The sections were then mounted onto gelatin-subbed slides, dehydrated, cleared in xylene, and coverslipped with Permount (Fisher, Pittsburgh, PA).

The cholera toxin labeling of thalamus was visualized with an antibody to the toxin. The sections were rinsed with $0.1 \mathrm{M}$ PBS with $0.3 \%$ Triton $\mathrm{X}-100$ and $0.34 \%$ sodium azide and treated with $0.1 \mathrm{M}$ glycine for $1 \mathrm{hr}$. The nonspecific binding was blocked with $3 \%$ normal rabbit serum (Vector Laboratories, Burlingame, CA) for $1 \mathrm{hr}$. The sections were then transferred into the primary antibody (goat anti-cholera toxin, 1:2000; List Biological Labs) with $3 \%$ normal rabbit serum at $4^{\circ} \mathrm{C}$ for $3 \mathrm{~d}$. The sections were rinsed again and placed in $\mathrm{Cy}$-2-conjugated rabbit anti-goat secondary antibody (Jackson ImmunoResearch, West Grove, PA) with $3 \%$ normal rabbit serum for $4 \mathrm{hr}$ in the dark. The sections were mounted, dried, cleared in xylene, and coverslipped with Krystalon (Diagnostic Systems, Gibbstown, NJ).

Bouton plotting and data analysis. Based on our own and others' studies of the location of normal ferret AI (Kelly et al., 1986; Phillips et al., 1988) and on our own anatomical and physiological studies on cross-modal ferrets (Pallas et al., 1990; Roe et al., 1990, 1992; Pallas and Sur, 1993), AI was defined as the region on the ectosylvian gyrus bounded by the anterior and posterior ectosylvian sulci, medial to the pseudosylvian sulcus (Fig. $1 A$ ). In each case, sections at a depth of 300-500 $\mu \mathrm{m}$ under the pia (layer 2/3) were selected for three-dimensional plotting of boutons using a camera lucida and Neurolucida software (Microbrightfield, Burlington, VT) under a Zeiss Axiophot microscope at a magnification of $400 \times$. The location and size of clusters of boutons were also recorded. Clusters were drawn by a blind observer and defined according to the presence of concentrated boutons and the relatively clear-cut borders of each cluster. The isolated axons and radiating fibers around the injection site were excluded from measures of cluster area. Boutons were sometimes found lateral to the AI border in the region known as AII, but these were excluded from the analysis.

To quantitatively describe the bouton distributions of each animal, polar plots of bouton distribution were constructed (Fig. 1B). The location of the injection site was defined as the plot origin. The angle and distance of individual bouton clusters relative to the injection site (polar coordinates) and their width and height were measured using Microbrightfield software. The horizontal axis $\left(0-180^{\circ}\right)$ was not corrected for section tilt on each slide in plots of individual cases, but was normalized for interanimal comparisons. In that case, a straight line was drawn from the top of the pseudosylvian sulcus (pss) extending to the top center of the suprasylvian sulcus to normalize the angular orientation of sections. This allowed direct, location-matched comparison of the bouton cluster distributions in AI of both normal and cross-modal animals. Other quantitative measures taken were bouton density (number of boutons per cluster area), the SD in the location of each bouton cluster compared with the location of all clusters (eccentricity, or how scattered the clusters were), the percent coverage or zone of influence of all boutons together [obtained by drawing a perimeter through the center of the outermost bouton clusters (Fig. $1 B$ ) and dividing that area by the area of $\mathrm{AI}]$, and the proportion of AI occupied by the clusters themselves (obtained by determining the area covered by each cluster, adding all cluster areas in each case, and calculating a percentage of the total area of AI). To compare cluster eccentricity between animals, polar plots from different cases were aligned by rotating the $y$-axis of each plot by the number of degrees necessary to match the orientation of the cluster vector closest to horizontal. Comparisons were made in each $90^{\circ}$ sector of the map to analyze spatial differences across the extent of AI. Statistical comparisons were done using a Pearson's correlation coefficient analysis or $t$ test as indicated, and all measurements were quoted as means \pm SE.

\section{RESULTS}

In the following, we report on how the qualitative features (laminar and tangential) of the horizontal connections revealed by the BDA injections differ in normal compared with cross-modal AI, followed by an account of the quantitative differences between the two groups. The BDA injections labeled numerous clusters of boutons within AI. In general, we found that the horizontal connectivity patterns in normal ferret AI are organized as they are in cats, in a strip corresponding to the isofrequency axis [which runs anteroposteriorly in ferrets (Kelly et al., 1986; Phillips et al., 1988), perpendicular to its orientation in cats]. However, the bouton clusters in the cross-modal cases were more uniformly arranged and were not confined to an anteroposterior strip, suggesting that the early visual inputs directed a reorganization of the normal pattern.

\section{Methodological considerations}

A potential source of artifact with any tracer injection is variability in the size and the depth of the tracer uptake zone. To overcome this drawback, we marked the tip of the injection micropipette at $500 \mu \mathrm{m}$ intervals so that depth of the pipette placement could be measured and thus controlled. The injections were also done slowly (over 15-20 min) to prevent brain injuries caused by pressure buildup. We did note that when the injection amount of BDA was $>200 \mathrm{nl}$ or when the depth of the injection pipette was close to layer 6 and the white matter, the anterograde labeling was very variable, and thus those cases were excluded from this study.

We did not attempt to analyze labeled terminals that were within $500 \mu \mathrm{m}$ of the injection site. There were two reasons for this: the high density of labeled processes and terminals close to the halo from the injection made it difficult to obtain an accurate impression of them, and also the local axonal projections may arise from a different class of axon collaterals (Ojima et al., 1991; Dalva and Katz, 1994), including inhibitory interneurons.

\section{Injection size and location}

The BDA injections were located approximately in the center of AI to facilitate comparison across animals. They consistently produced good anterograde label in AI. Labeled axons were readily recognized even in the close vicinity of the injection site. In each case, there was necrosis at the core region of the injection site and a halo zone a short distance around it. Only in the core region was the labeling obscured by the dark reaction product. In Figure $2 A$ a typical injection site is shown, and neuronal fibers can be seen streaming in an anterograde direction away from the site. These BDA injections produced label in a series of patches or clusters at a distance from the injection site (Fig. 2B). The clusters varied in the density of their neuronal processes as well as their bouton density (Fig. $2 C$ ). Clusters were more dense near the sulcal borders. In addition to the labeled axonal terminals, a few retrogradely labeled somata were also seen (Fig. 2B), although they tended to lie outside of the bouton clusters.

Care was taken to make comparably sized BDA injections in all animals to ensure that any differences seen between normal and cross-modal cases were not caused by this variable. To ensure that the variation was similar in normal and cross-modal groups, the core of the injection as well as the diameter of the halo of label surrounding the injection site were measured (Table 1). The injection sites were small (mean core diameter of $417 \pm 30.6 \mu \mathrm{m}$ in normal animals and $366 \pm 33.7 \mu \mathrm{m}$ in cross-modal animals, and a mean halo diameter of $565 \pm 60.1 \mu \mathrm{m}$ in normal animals compared with $507 \pm 34.7 \mu \mathrm{m}$ in cross-modal animals), and did not differ significantly between normal and experimental animals (for core size, $p=0.27$; for halo size, $p=0.420$ ). Furthermore, injection size was not significantly correlated with the number of labeled bouton clusters within each group (see below) $(r=0.089$ for normal and 0.268 for cross-modal cases). Thus, injection size was unlikely to be a factor in the differences seen between normal and cross-modal cases. We did note that with very large injections (>200 $\mathrm{nl}$ of BDA and $1000 \mu \mathrm{m}$ diameter injection sites), the anterograde label was distributed more widely, as would be expected in normal animals if the injection crossed many isofrequency lines (Wallace and Bajwa, 1991). Such cases were not included in this study. 

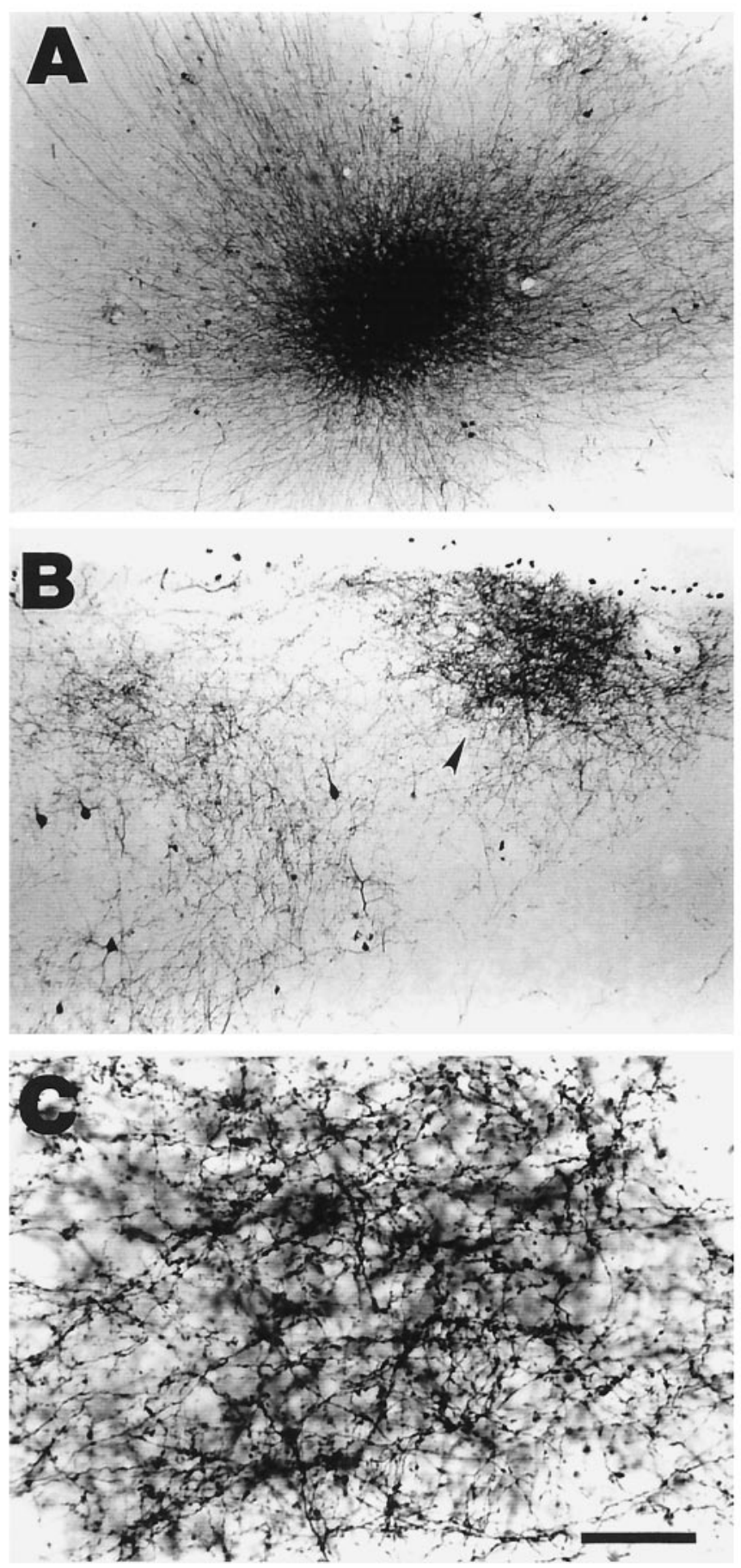

Figure 2. BDA injections were made into layer $2 / 3$ of AI in adult ferrets. $A$, An example of an injection site in a tangential section through AI. Note the relatively small and densely packed appearance of the halo and the fibers exiting the center. $B$, The BDA injections resulted in the labeling of several clusters of axon terminals in AI. This micrograph shows two such clusters, one very dense (arrowhead) and the other more sparse. C, At higher magnification, individual boutons within a patch can be seen. The locations of these boutons were mapped at high magnification and used for data analysis. Scale bar: $A, 300 \mu \mathrm{m} ; B, 150 \mu \mathrm{m} ; C, 40 \mu \mathrm{m}$.

\section{Relationship between lesion size and retinal axon rerouting}

A second technical variable that required consideration was the fact that the amount of retinal axon rerouting into the MGN depends on the size of the neonatal lesions (Angelucci et al., 1997, 1998). For this reason, lesions were reconstructed and ranked as large $(>75 \%$ of the midbrain absent), medium (50$75 \%$ of the midbrain absent), or small ( $<50 \%$ missing). Of the seven cross-modal ferrets used in this study, two had a large lesion (9836 and 9869), four had a medium lesion (9834, 9837, 9865, and 9823), and one had a small lesion (9819). When the pattern of anterograde retino-MGN label from eye injections was analyzed, we found that $100 \%$ of the cross-modal ferrets had retino-MGN axons, and four of seven had numerous such axons. Retinal axons were very rarely seen in MGN of normal animals; in the course of several investigations using intraocular cholera toxin injections, we have seen retino-MGN axons in $<10 \%$ of animals $(n=57)$, and then only one axon was observed that appeared to have strayed slightly from the medial border of the LGN (Pallas and Moore, 1997).

Although the cases used in this study varied in lesion size and extent of cross-modal rewiring, the lack of multiple cases at each lesion size prevented us from firmly relating any observed differences in horizontal connectivity patterns to this variable. It is possible that with a larger study involving a wider variety of lesion sizes an effect could be seen, and this would be an interesting avenue for further investigation.

\section{Tangential distribution of horizontal connections}

The observed differences in the tangential distribution of bouton clusters between normal and cross-modal cases were analyzed qualitatively with respect to their number and mediolateral or anteroposterior location. The distribution of clusters in the tangential plane was clearly different in normal animals as compared with cross-modal animals. The clusters in normal animals were distributed mainly in strips extending anteriorly and posteriorly away from the injection site up to the anterior ectosylvian and posterior ectosylvian sulci (Fig. 3A). The anteroposterior axis corresponds to the isofrequency axis of the tonotopic map in these animals (Kelly et al., 1986; Kelly and Judge, 1994). However, in cross-modal animals, in addition to those axons extending along the isofrequency axis, the injections also labeled axons that extended medially, forming bouton clusters in medial AI (Fig. $3 B$ ). Along with bouton clusters located outside of anteroposterior strips, we noted that there were more clusters in cross-modal than normal AI, and they were noticeably more widespread in their distribution pattern when compared with the bouton clusters in normal AI.

To illustrate cluster distribution and confirm our light microscopic observations, the locations of all labeled boutons within layer 2/3 were mapped at high magnification $(400 \times)$. In Figure 4, bouton distributions in one representative tangential section are shown for four normal and four cross-modal cases. In the reconstructions from normal animals (Fig. 4, top), it can be seen that the BDA-labeled axonal arbors extended mainly anteriorly and posteriorly from the injection site and formed patches distributed along the anteroposterior axis. Clusters could be identified and followed through several serial tangential sections.

In contrast to normal cases, in cross-modal cases the bouton clusters were arranged in a semicircular pattern around the injection site (Fig. 4, bottom). In all seven of the cross-modal animals used in this experiment, the clusters were distributed in this semicircular pattern, whereas in normal animals the pattern was elongated. The presence of bouton clusters at the medial crown of the ectosylvian gyrus was in striking contrast to what was seen in normal AI, and in addition to being distributed more 
Table 1. Tracer injection and patch distribution ${ }^{a}$

\begin{tabular}{|c|c|c|c|c|c|c|}
\hline Animals & Lesion & $\mathrm{BDA}(\mathrm{nl})$ & $\begin{array}{l}\text { Size of injection } \\
\text { site }(\mu \mathrm{m})\end{array}$ & $\begin{array}{l}\text { Number of } \\
\text { patches }\end{array}$ & $\begin{array}{l}\text { Patch diameter } \\
(\mu \mathrm{m})\end{array}$ & $\begin{array}{l}\text { Distance to in- } \\
\text { jection center } \\
(\mu \mathrm{m})\end{array}$ \\
\hline 9814 & No & 100 & $665 \times 380$ & 15 & $1014.0 \pm 72.12$ & $1919.7 \pm 182.35$ \\
\hline 9820 & No & 100 & $285 \times 238$ & 10 & $758.3 \pm 67.39$ & $2146.1 \pm 332.61$ \\
\hline 9840 & No & 100 & $475 \times 285$ & 8 & $829.7 \pm 95.23$ & $1811.7 \pm 206.01$ \\
\hline 9847 & No & 100 & $570 \times 285$ & 16 & $712.1 \pm 47.41$ & $2020.8 \pm 206.76$ \\
\hline 9849 & No & 50 & $475 \times 427$ & 6 & $506.0 \pm 27.06$ & $2205.0 \pm 342.12$ \\
\hline 9864 & No & 100 & $475 \times 380$ & 8 & $770.9 \pm 106.4$ & $2283.6 \pm 394.31$ \\
\hline $9864 \mathrm{R}^{b}$ & No & 100 & $525 \times 380$ & 9 & $718.1 \pm 69.67$ & $2436.6 \pm 240.26$ \\
\hline Mean & & & $\begin{array}{l}498 \times 339 \\
(417.6 \pm 30.61)\end{array}$ & $10.3 \pm 1.43$ & $790.4 \pm 30.89$ & $2090.4 \pm 97.01$ \\
\hline 9819 & LVCxSCxBICx & 100 & $428 \times 285$ & 14 & $982.0 \pm 57.59$ & $2536.1 \pm 174.58$ \\
\hline 9823 & LVCxSCxBICx & 150 & $380 \times 238$ & 18 & $644.8 \pm 53.78$ & $1916.3 \pm 113.64$ \\
\hline 9834 & LVCxSCxBICx & 150 & $475 \times 380$ & 16 & $953.5 \pm 102.71$ & $1616.2 \pm 127.49$ \\
\hline 9836 & R/LVCxSCxBICx & 50 & $665 \times 237$ & 17 & $608.8 \pm 30.18$ & $2663.7 \pm 236.24$ \\
\hline 9837 & LVCxSCxBICx & 100 & $332 \times 285$ & 17 & $834.5 \pm 54.44$ & $1728.5 \pm 161.87$ \\
\hline 9865 & LVCxSCxBICx & 100 & $570 \times 380$ & 19 & $904.9 \pm 73.57$ & $1693.5 \pm 140.62$ \\
\hline 9869 & LVCxSCxBICx & 100 & $285 \times 190$ & 16 & $798.2 \pm 55.99$ & $2085.0 \pm 227.73$ \\
\hline Mean & & & $448 \times 285$ & $16.7 \pm 0.61$ & $812.1 \pm 26.80$ & $2018.3 \pm 72.33$ \\
\hline
\end{tabular}

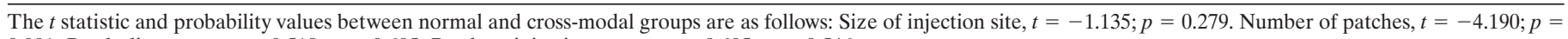
0.001 . Patch diameter, $t=-0.518 ; p=0.605$. Patch to injection center, $t=0.605 ; p=0.546$.

${ }^{a}$ All numbers are presented as mean $\pm \mathrm{SE}$ in this table.

${ }^{b}$ Right hemisphere of ferret 9864.

widely, the clusters also appeared more numerous. Some clusters in both groups extended lateral to the top of the pseudosylvian sulcus into what was probably the secondary auditory area AII (Fig. 4 bottom, top left) (Pallas et al., 1990; Pallas and Sur, 1993), but these were not included in further analyses.

Figure 5 shows polar plots of the three-dimensional extent of the bouton distributions of the labeled clusters in normal (Fig. 5, top) and cross-modal (Fig. 5, bottom) cases, corresponding to the reconstructions shown in Figure 4. These plots were made by drawing a perimeter around the centers of the outermost bouton clusters (Fig. 1), and they illustrate the overall extent of coverage of auditory cortex by horizontal connections; i.e., their zone of influence or coverage area. The polar plots of the coverage areas in normal animals were stripe-like and elongated anteroposteriorly along the isofrequency axis of the tonotopic map. In contrast, in cross-modal animals the coverage areas extended over a larger area of AI and were not strip-like, but were more circular or isotropic, with a rosette or fan shape.

The cluster distributions from all seven normal and seven cross-modal animals are summarized in Figure 6. The labeled bouton clusters in normal AI were arranged in an elongated fashion on either side of the injection site in all cases. Within the anterior half of AI, the population of clusters was centered on a vector of $163^{\circ} \pm 6.6$, and in the posterior half it was centered at $14.8^{\circ} \pm 7.5$. Most bouton clusters in normal AI were located within these more anterior and posterior sectors of the polar plot, rather than in the medial (centered on $90^{\circ}$ ) and lateral (centered on $270^{\circ}$ ) sectors. There were no clusters in the sectors at $60-90^{\circ}$ or $300-330^{\circ}$ and very few at $180-300^{\circ}$. However, in the crossmodal cases all of these regions contained several clusters.

\section{Quantitative analysis of bouton clusters}

To characterize the distributions of labeled bouton clusters in both groups quantitatively, several measures were taken. To an- alyze the individual clusters, cluster size (diameter $=$ mean of height and width) and shape (elongation factor, as determined by the ratio of height to width) were measured. There were no significant differences between the normal and cross-modal groups in either cluster size (Table 1, Fig. $7 A$; mean of normals, $790.4 \pm 30.89 \mu \mathrm{m}$ and mean of cross-modals, $812.1 \pm 26.80 \mu \mathrm{m}$; $p=0.6$ ) or shape (Fig. $7 B ; 1.6 \pm 0.05$ in normal and $1.5 \pm 0.04$ in cross-modal; $p=0.4$ ), suggesting that the early visual inputs have no effect on the general morphology of bouton clusters. However, the visual input did have a significant effect on the number of bouton clusters contained within AI (Table 1, Fig. $7 C$; normal mean, $10.3 \pm 1.43$; cross-modal mean, $16.7 \pm 0.61 ; p=0.001)$.

To analyze this difference further, a comparison of the distribution of bouton clusters was made between normal and crossmodal cases. Bouton density within the clusters was averaged and found to be similar in the two groups $(3.26 \pm 0.91$ boutons $/ 1000$ $\mu \mathrm{m}^{2}$ in normals and $3.72 \pm 0.63$ in cross-modal cases; $p=0.7$ ). We found that the distribution of the distances of individual clusters to the center of the injection site was also similar in both groups (Fig. $7 D$, Table $1 ; p=0.5$ ), demonstrating that although the clusters are more numerous in cross-modal than in normal AI, they maintain a similar lateral extent. However, this is not true when the entire zone of influence is considered. Because of the widespread nature of the clusters in the cross-modal cases, they have a much greater zone of influence (Fig. 7E; normal, $27.2 \pm$ $4.51 \%$; cross-modal, $52.9 \pm 6.22 \% ; p=0.001$ ) compared with normal AI. Consistent with this, a measure of the proportion of AI occupied by the clusters themselves shows that they occupied significantly less of AI in normal than in cross-modal cases (7.2 \pm $1.36 \%$ in normals vs $14.8 \pm 2.60 \%$ in cross-modals; $p=0.02)$. Thus, the horizontal projections from a given locus in AI can influence a larger extent of AI in cross-modal animals, even 

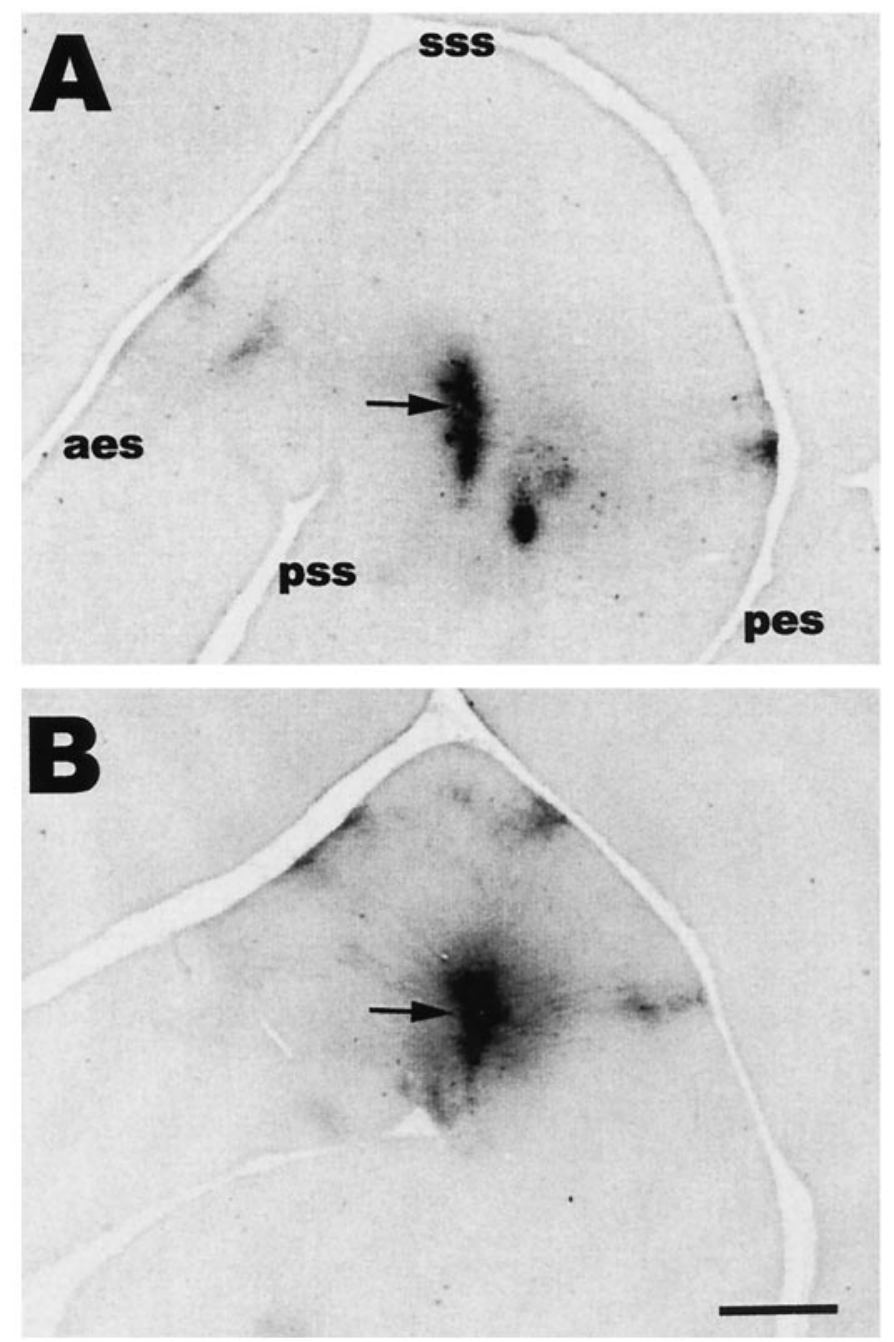

Figure 3. Digitized micrograph of a tangential section through layer $2 / 3$ of AI, showing the pattern of bouton clusters arising from long range horizontally projecting axons in representative normal $(A)$ and crossmodal $(B)$ animals. The arrows indicate the injection sites. Anterior is to the left, and medial is up in this and the other figures. Scale bar, $1 \mathrm{~mm}$.

though the radius of influence (cluster distance from the injection site) does not change.

It is clear from the camera lucida reconstructions of labeled boutons that the bouton clusters are less likely to lie on the anteroposterior, isofrequency axis in cross-modal animals. To quantify the degree to which bouton clusters varied away from the anteroposterior axis, a cluster eccentricity from this axis was calculated. This calculation of how far the labeled bouton clusters scattered from the axis was made by measuring the SD of clusters from their anteroposterior axis (eccentricity). The mean scatter was $884.5 \pm 61.87 \mu \mathrm{m}$ in cross-modal animals, which was significantly greater than the $619.1 \pm 60.82 \mu \mathrm{m}$ extent of scatter in normal animals $(p=0.005$; Fig. $7 F)$.

In sum, these quantitative measures confirm the qualitative impressions presented above. The labeled clusters of boutons in cross-modal AI, although of similar size and shape, and comparable distance from the injection site, were more widely distributed than in normal animals, and were scattered further from the

\section{Normal}
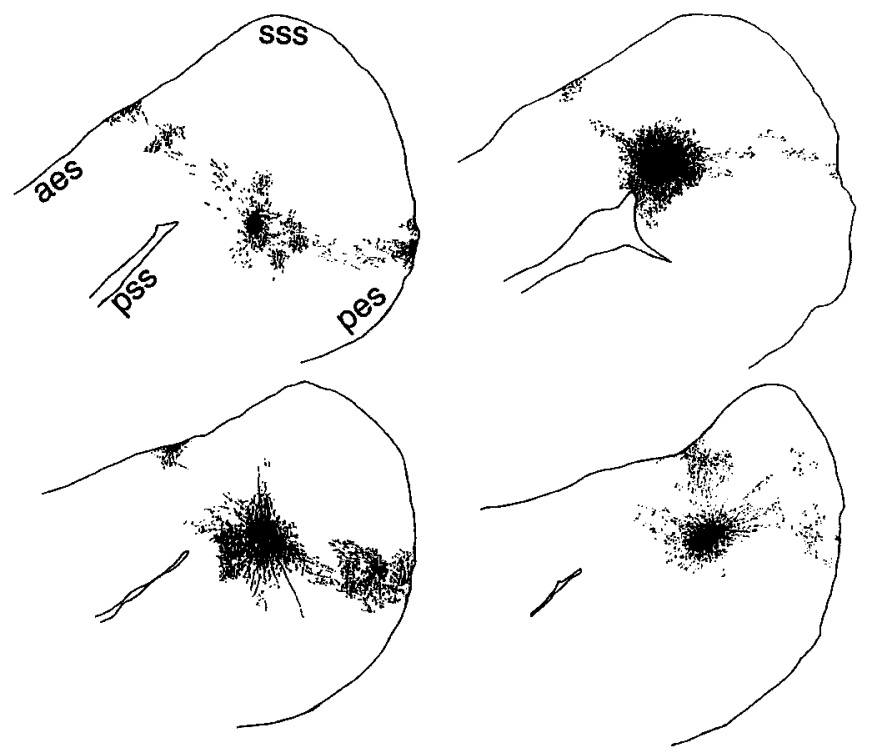

Cross-modal

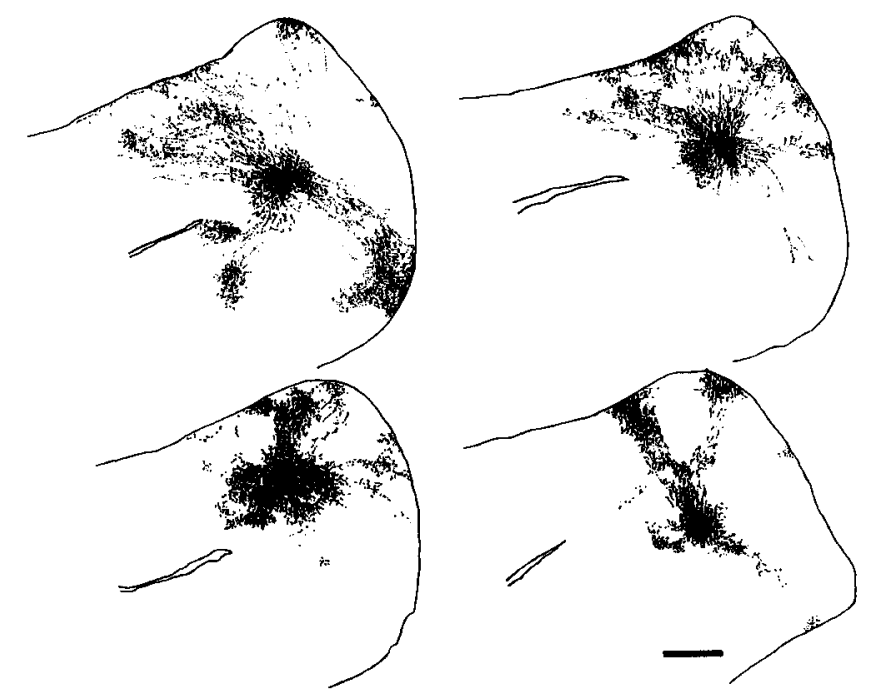

Figure 4. Reconstruction of the bouton distributions in a representative tangential section through layer $2 / 3$ from four normal (top) and four cross-modal (bottom) cases after BDA injections in AI. Each dot represents a single bouton. The BDA-labeled axonal arbors in normal ferrets were distributed mainly in an anteroposterior direction, along the same axis as the isofrequency domains. The case shown in the bottom right was the most atypical in that the boutons were arranged more medially than in the other cases. In cross-modal AI, the axonal projections and thus the bouton clusters were more widely distributed than in normal AI and were more likely to be found in the medial part of AI. In one case, there are two clusters ventral to the pseudosylvian sulcus which are likely located in AII rather than AI. Scale bar, $1 \mathrm{~mm}$.

isofrequency axis, where they were in a position to influence a larger proportion of the cortical circuitry within AI.

\section{Laminar distribution of horizontal connections}

The pattern of horizontal connectivity in AI was analyzed in both the laminar and tangential planes. Although all brains used in this study were sectioned in the tangential plane, the corresponding Nissl-stained sections allowed determination of the approximate 


\section{Normal}
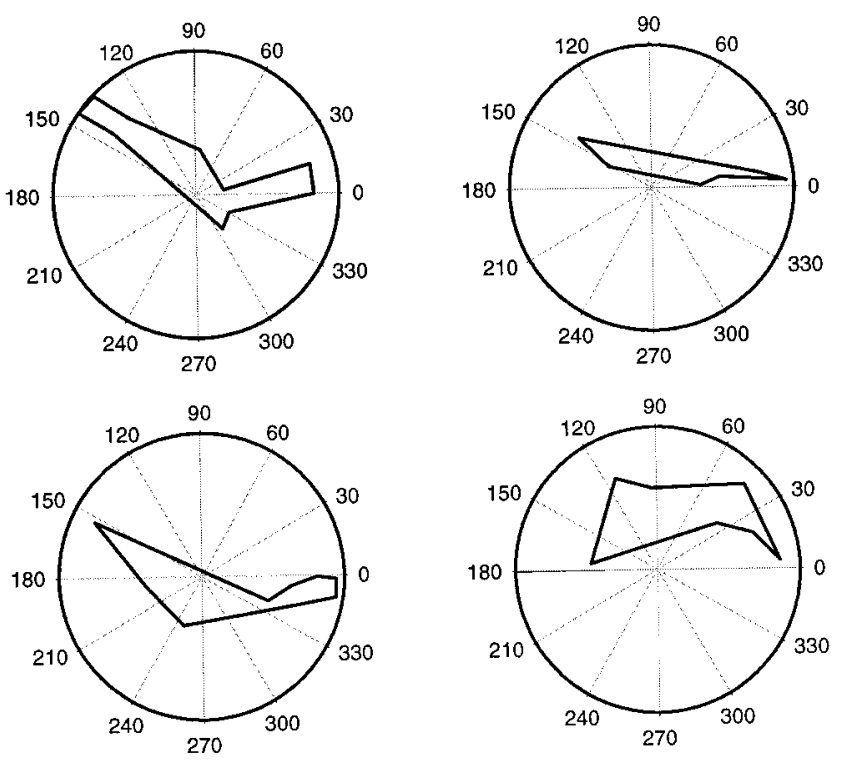

\section{Cross-modal}
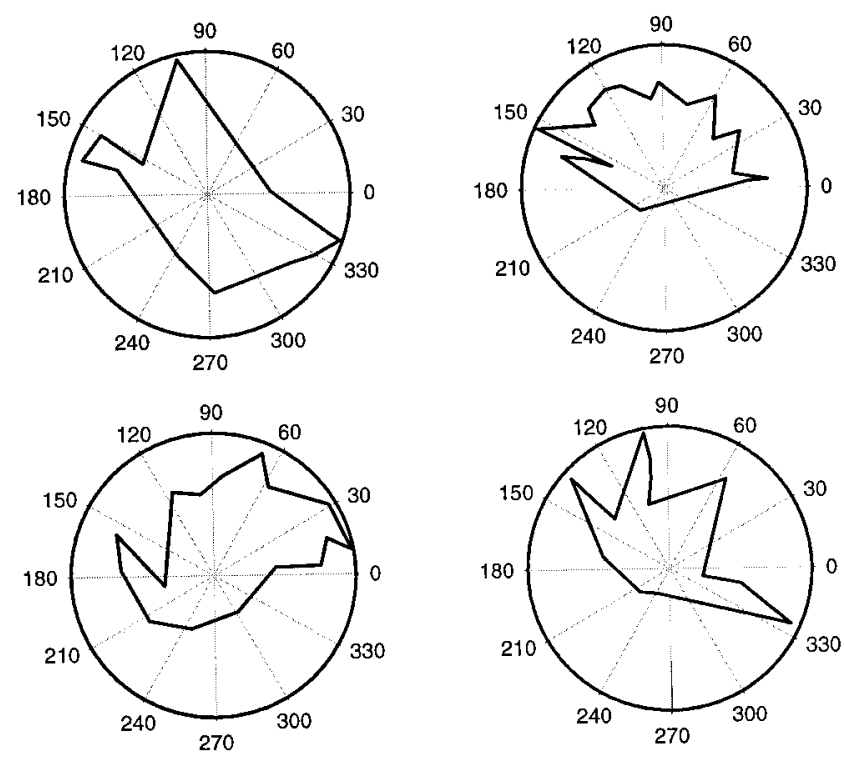

Figure 5. Polar plots showing the coverage area of the labeled boutons from four normal (top) and four cross-modal (bottom) cases, corresponding to those in Figure 4. The bouton clusters covered an elongated strip extending anteroposteriorly in normal animals, but in cross-modal cases covered a wider, more circular area and extended mediolaterally as well as anteroposteriorly, often resembling a rosette-like pattern.

laminar location of neuronal fibers and boutons by calculating their position in relation to the granule cell layer. BDA injections were targeted on layer $2 / 3$ in AI, at a depth of 300-500 $\mu \mathrm{m}$ from the cortical surface, and thus the neuronal processes leaving the injection site originated primarily from those layers. However, in three normal and four cross-modal cases, a few clusters of horizontal connections could also be observed in the deeper layers. Bouton clusters also sometimes appeared in the secondary auditory cortical area, AII. In this study only boutons in layers $2 / 3$ of area AI were analyzed. Occasionally, some of the labeled axons entered the white matter, but were never observed extending into the contralateral hemisphere. No apparent differences in the laminar extent of BDA-labeled fibers were consistently observed between normal and cross-modal cases, within the limitations presented by tangential reconstructions. Further information could be gathered on this topic by repeating this study and sectioning the tissue in the coronal plane.

\section{DISCUSSION}

The results of this study show that the characteristic horizontal connections between auditory cortical neurons can be altered by early anomalous visual input, and become less elongated than normal, deviating substantially from the isofrequency axis. In addition to the change in the pattern of horizontal connections, their number and zone of influence were increased. Therefore, our results support the hypothesis that local horizontal connections in cross-modal AI are susceptible to instruction from the visually driven pattern of activity in thalamocortical inputs during postnatal development. Because we have not altered the thalamocortical pathway in this paradigm, we suggest that the identity of the thalamic nucleus carrying the visual information does not influence this process, or at least that it cannot override the sensory modality-based instructions for constructing intrinsic cortical circuits.

\section{Horizontal connections in normal Al are oriented along the anteroposterior axis}

In primary visual cortex, long distance intracortical collaterals of pyramidal neurons link together neurons responding to similar stimulus features in a manner independent of their location (for review, see Rockland, 1998). This general organizational feature has also been seen in primary auditory cortex of cats and ferrets, where horizontal connections link neurons along an isofrequency domain (Reale et al., 1983; Matsubara and Phillips, 1988; Ojima et al., 1991; Wallace et al., 1991; Clarke et al., 1993).

Our results show that the pattern of horizontal connections in normal ferrets is similar to that in cats, except that the trajectory of bouton clusters was oriented anteroposteriorly, roughly perpendicular to its orientation in cats (Reale et al., 1983). However, the tonotopic map in ferrets is also perpendicular to that in cats, with high frequencies represented medially and low frequencies laterally (Kelly et al., 1986; Phillips et al., 1988). Thus, the horizontal connections are oriented as would be expected if their function in ferrets and cats is to link neurons with similar sound frequency tuning. In the context of this study, we did not determine whether there was any restriction of connections according to binaural properties; this would be an interesting question for a combined anatomical/physiological study.

A previous report on horizontal connectivity patterns in normal ferret AI (Wallace and Bajwa, 1991) proposed that horizontal collaterals link neurons with both similar and different frequency tuning, unlike in cats. These results raised the interesting possibility that a species difference exists and that auditory stimulus features other than frequency are linked in an organized fashion in ferrets but not cats. However, our data on horizontal connectivity in normal ferret AI differ with this interpretation, because we found bouton clusters to be very strongly biased along the anteroposterior, isofrequency axis, and almost completely excluded from other regions. This difference is probably the result of a difference in methodology, because the injections in this study were smaller and perhaps more superficial than those in the 


\section{Normal}

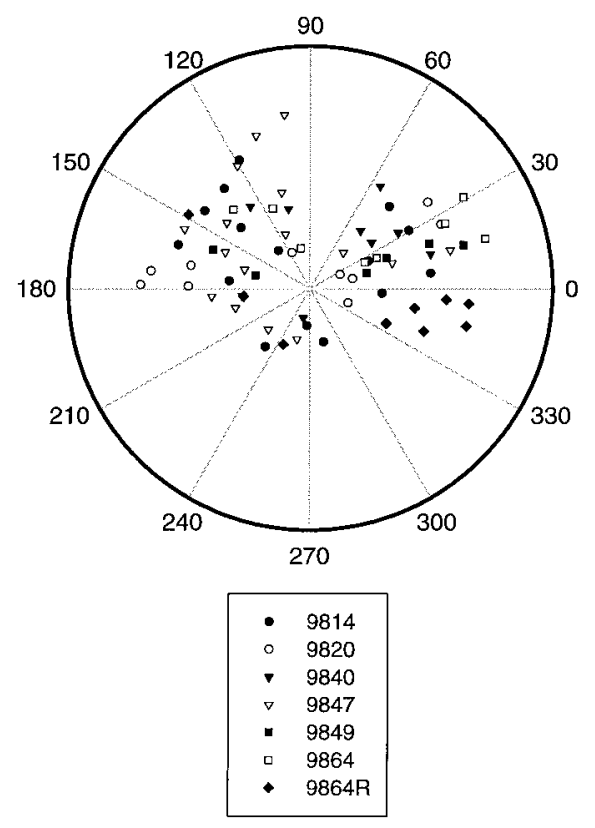

\section{Cross-modal}

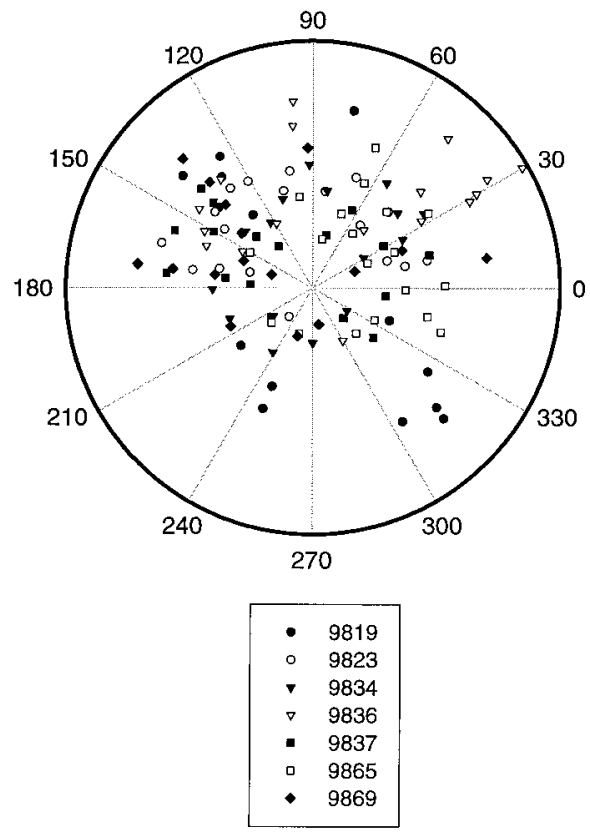

Figure 6. Combined polar plots showing the locations of the centers of all bouton clusters from seven normal cases (left) and seven crossmodal cases (right). To align the plots from different animals, the horizontal axis of the plot $\left(0-180^{\circ}\right)$ was aligned with the axis of the pseudosylvian sulcus in each case (Fig. 1), and the polar coordinates of the center of each bouton cluster were recorded. Each case is represented by its own symbol. The clusters in normal animals were anisotropically arranged and were preferentially located within anterior and posterior sectors. In contrast, the labeled bouton clusters in cross-modal ferrets were arranged more isotropically and were located in all sectors. other study. Larger injections would be expected to label more isofrequency laminae.

\section{Horizontal connections in cross-modal Al are arranged in a semicircular pattern}

We found that the pattern of long distance, horizontal projections in AI with early visual input was distinctly different from that in normal AI. Bouton clusters were arranged in a semicircle around injection sites, rather than being arranged anisotropically along the anteroposterior axis. The clusters were also greater in number and thus in the proportion of AI that they occupied. Despite these differences in number and pattern, the shape and size of the clusters was unaffected by visual input, and these characteristics are perhaps outside of afferent activity-based control. Our interpretation of these data are that the visually driven activity of thalamic inputs, and not their anatomical source, controls the organization of intracortical circuitry. This could occur via Hebbian mechanisms, based on correlated activation of neighboring retinal neurons or neurons with similar visual response properties.

In cat and ferret visual cortex, long distance axon collaterals are initially widespread, and bouton clusters only begin to develop postnatally (Rockland and Lund, 1982; Callaway and Katz, 1990; Dalva and Katz, 1994; Durack and Katz, 1996). Spontaneous activity is required for early cluster formation, whereas later stages of refinement are dependent on visually driven activity (Callaway and Katz, 1991; Löwel and Singer, 1992; Ruthazer and Stryker, 1996). The development of horizontal connectivity in ferret or cat auditory cortex has not been studied, although callosal connections in cat auditory cortex show a similar, early diffuse developmental pattern as in cat V1 (Feng and Brugge, 1983). There are several reasons to conclude that the circular arrangement of bouton clusters that we observed is driven by the visual inputs, and not by a lack of normal auditory input to AI in cross-modal animals. One is that our studies of callosal connections in deafened and cross-modal ferrets (Pallas et al., 1999) show that the cross-modal callosal connectivity pattern is distinct from the pattern in both the normal and deaf groups. Second, if the location of clusters outside of the isofrequency axis resulted from a lack of correlated input activity, it might be expected that they would remain only partially refined, as in deprived visual cortex. Instead, the clusters in the cross-modal animals actually looked more dense and compact than in normal animals. Furthermore, the number of clusters was greater than normal in the cross-modal cases. It is difficult to envision how a relaxation of cluster refinement mechanisms would result in an increased number of more refined clusters. Finally, our preliminary studies indicate that horizontal connections in deafened animals are diff use and unorganized, suggesting that in the cross-modal animals the early anomalous visual inputs are driving the formation of specific projections from intracortically projecting pyramidal neurons (Gao et al., 1999; Pallas and Gao, 1999).

The functional role of the long distance projections in crossmodal AI cannot be determined from our anatomical data. There is a retinotopic map in cross-modal AI, and neurons tuned to visual stimulus orientation are also found there (Roe et al., 1990, 1992). The altered horizontal connectivity pattern may be responsible in part for the creation of these visual response properties in auditory cortex. An intriguing possibility is that the bouton clusters in cross-modal AI are organized as in visual cortex, where neurons with similar response properties, such as orientation tuning, are connected in an isotropic pattern across the retinotopic map. Although there is some suggestion that neurons in cross-modal AI with similar orientation tuning are clustered (Sharma et al., 1996), it is unknown whether they are arranged in pinwheel fashion as in visual cortex. The resemblance seen between the arrangement of bouton clusters in AI and those found in normal ferret visual cortex (Weliky and Katz, 1994; Durack and Katz, 1996; Ruthazer and Stryker, 1996) is suggestive of a similar function. Alternatively, there may be a different substrate for bouton cluster organization in cross-modal AI that 


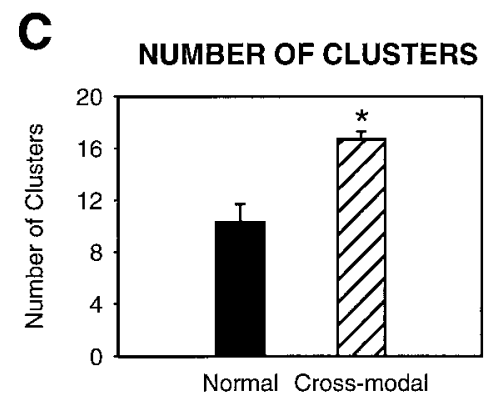

Figure 7. Quantitative comparison of the bouton clusters in both normal (dark bars) and cross-modal (striped bars) animals. Asterisks indicate significant differences. $A, B$, Comparisons at the level of individual clusters. $A$, Cluster size (average of height and width) was similar in both groups. $B$, Cluster shape as measured by elongation factor (major axis of patch divided by minor axis) was also not significantly different between the two groups. $C-F$, Comparisons at the level of bouton cluster patterns. $C$, The number of clusters was significantly greater in cross-modal than normal AI. $D$, Cluster distance distribution. The distance of bouton clusters from the center of the injection site in both normal and crossmodal animals was similar. $E$, The area which was covered by bouton clusters (zone of influence) was also significantly larger in the cross-modal cases. $F$, Amount of scatter (SD) of bouton clusters away from the horizontal, isofrequency axis of the polar plots. This was a measure of the tendency of clusters to be more widely distributed in cross-modal than normal animals. There was a significant difference between the two groups.
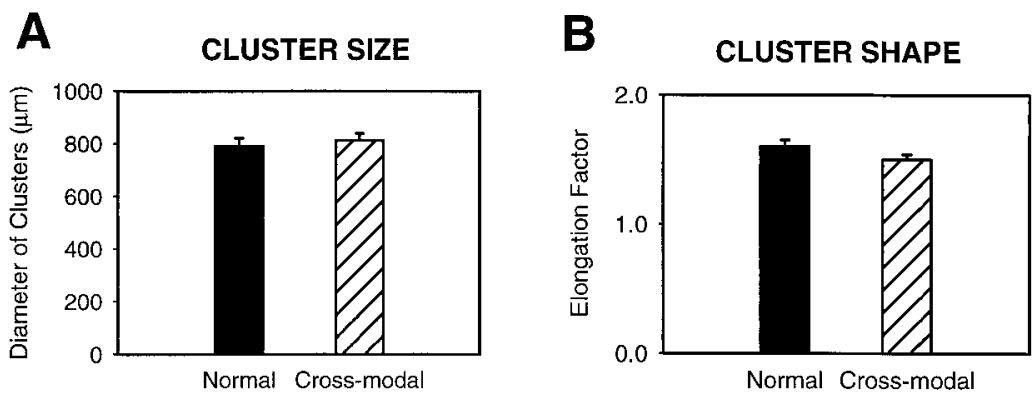

D

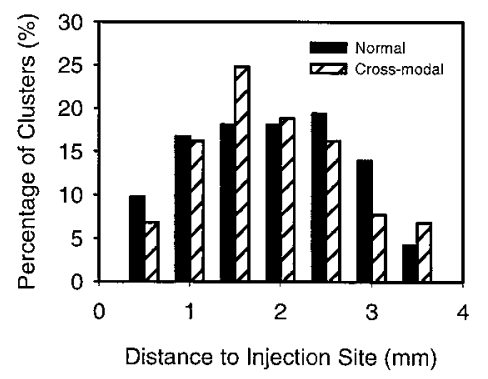

E

ZONE OF INFLUENCE

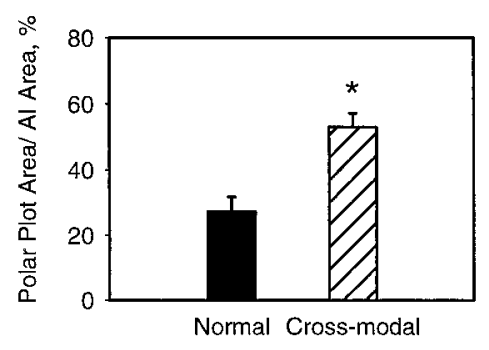

$\mathbf{F}$

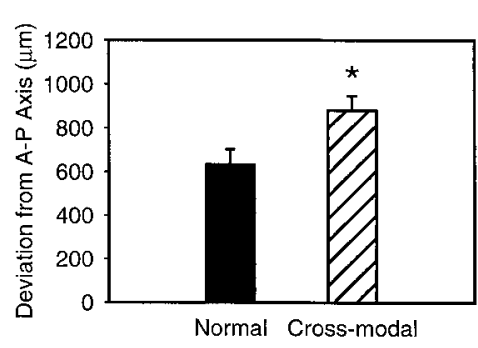

is also roughly isotropic in pattern. Our future efforts will be directed at answering this question.

\section{Implications for cortical specification}

Cortical areal specification refers to a stepwise process whereby the fate ("identity") of a cortical region is progressively restricted. Different steps are likely to be controlled by different factors. There is substantial evidence that thalamocortical afferents play a role in establishing some aspects of regional identity. Several molecular markers are found only in certain cortical regions (Levitt, 1984; Arimatsu et al., 1992; Cohen-Tannoudji et al., 1994; Paysan et al., 1994), and in at least one case marker expression seems to be triggered by thalamic input (Levitt et al., 1997), but a causal relationship between marker expression and areal specification is difficult to establish. It is clear that there are detailed recognition mechanisms guiding thalamic axons to the proper cortical region (Ghosh et al., 1990; Suzuki et al., 1997; Castellani et al., 1998; Inoue et al., 1998; Mann et al., 1998), suggesting that thalamocortical axons may instruct regional identity. This instruction could depend on the source of the thalamocortical axons, their activity pattern, or both. The results of this study, by showing that auditory thalamic axons carrying visual information can reorganize horizontal projections, strongly suggest that the organization of intrinsic cortical connections depends not on the source of thalamic inputs but on their modality-dependent activity patterns. The results further suggest that activity in this case is not merely permissive but instructive (Crair, 1999).
Clinical evidence relevant to this issue includes the observation that in blind and deaf patients, the "unused" cortical area can be taken over and used by a different sensory modality (Neville et al., 1983; Sadato et al., 1996; Cohen et al., 1997; Kujala et al., 1997; Lessard et al., 1998). Cross-modal plasticity has also been demonstrated in other animal models (Rebillard et al., 1977; Heil et al., 1991; Rauschecker, 1995), but it has not been clear in each case whether the novel inputs reorganize cortical circuitry during development or whether they simply take advantage of existing circuits. Furthermore, it is not known whether the invasion and reorganization process is dependent on the pattern of activity or the identity of the thalamocortical afferents. Our results suggest that activity-dependent control over cortical circuit formation by thalamocortical afferents, based on correlated activity within neighboring regions of the retina, may explain a number of these observations. Furthermore, it may provide a general mechanism for cortical regionalization at the circuit level of organization. Such an afferent-driven organization rule would have broad implications for both the development and the evolution of mammalian sensory cortex.

\section{REFERENCES}

Angelucci A, Clascá F, Bricolo E, Cramer KS, Sur M (1997) Experimentally induced retinal projections to the ferret auditory thalamus: development of clustered eye-specific patterns in a novel target. J Neurosci 17:2040-2055.

Angelucci A, Clascá F, Sur M (1998) Brainstem inputs to the ferret 
medial geniculate nucleus and the effect of early deafferentation on novel retinal projections to the auditory thalamus. J Comp Neurol 400:417-439.

Arimatsu Y, Miyamoto M, Nihonmatsu I, Hirata K, Uratani Y, Hatanaka Y, Takiguchi-Hayashi K (1992) Early regional specification for a molecular neuronal phenotype in the rat neocortex. Proc Natl Acad Sci USA 89:8879-8883.

Bosking WH, Zhang Y, Schofield B, Fitzpatrick D (1997) Orientation selectivity and the arrangement of horizontal connections in tree shrew striate cortex. J Neurosci 17:2112-2127.

Callaway EM, Katz LC (1990) Emergence and refinement of clustered horizontal connections in cat striate cortex. J Neurosci 10:1134-1153.

Callaway EM, Katz LC (1991) Effects of binocular deprivation on the development of clustered horizontal connections in cat striate cortex. Proc Natl Acad Sci USA 88:745-749.

Castellani V, Yue Y, Bolz J (1998) Dual action of a ligand for eph receptor tyrosine kinases on specific populations of axons during the development of cortical circuits. J Neurosci 18:4663-4672.

Catalano SM, Shatz CJ (1998) Activity-dependent cortical target selection by thalamic axons. Science 281:559-562.

Clarke S, de Ribaupierre F, Rouiller EM, de Ribaupierre Y (1993) Several neuronal and axonal types form long intrinsic connections in the cat primary auditory cortical field (AI). Anat Embryol (Berl) 188:117-138.

Cohen LG, Celnik P, Pascual-Leone A, Cornwell B, Faiz L, Dambrosias J, Honda M, Sadato N, Gerloff C, Catala MD, Hallett M (1997) Functional relevance of cross-modal plasticity in blind humans. Nature 389:180-186.

Cohen-Tannoudji M, Babinet C, Wassef M (1994) Early determination of a mouse somatosensory cortex marker. Nature 368:460-463.

Crair MC (1999) Neuronal activity during development: permissive or instructive? Curr Opin Neurobiol 9:88-99.

Dalva MB, Katz LC (1994) Rearrangements of synaptic connections in visual cortex revealed by laser photostimulation. Science 265:255-258.

Durack JC, Katz LC (1996) Development of horizontal projections in layer $2 / 3$ of ferret visual cortex. Cereb Cortex 6:178-183.

Feng JZ, Brugge JF (1983) Postnatal development of auditory callosal connections in the kitten. J Comp Neurol 214:416-426.

Gao W-J, Moore DR, Pallas SL (1999) Bilateral cochlear ablation in neonatal ferrets prevents refinement of horizontal connectivity in primary auditory cortex. Soc Neurosci Abstr 25, in press.

Ghosh A, Antonini A, McConnell SK, Shatz CJ (1990) Requirement for subplate neurons in the formation of thalamocortical connections. Nature 347:179-181.

Gilbert CD, Wiesel TN (1979) Morphology and intracortical projections of functionally characterised neurones in the cat visual cortex. Nature 280:120-125.

Gilbert CD, Wiesel TN (1983) Clustered intrinsic connections in cat visual cortex. J Neurosci 3:1116-1133.

Gilbert CD, Wiesel TN (1989) Columnar specificity of intrinsic horizontal and corticocortical connections in cat visual cortex. J Neurosci 9:2432-2442.

Heil P, Bronchti G, Wollberg Z, Scheich H (1991) Invasion of visual cortex by the auditory system in the naturally blind mole rat. NeuroReport 2:735-738.

Imig TJ, Reale RA, Brugge JF, Morel A, Adrian HO (1986) Topography of cortico-cortical connections related to tonotopic and binaural maps of cat auditory cortex. In: Two hemispheres-one brain: functions of the corpus callosum (Leporé F, Ptito M, Jasper HH, eds) pp 103-115. New York: Alan R. Liss.

Inoue T, Tanaka T, Suzuki SC, Takeichi M (1998) Cadherin-6 in the developing mouse brain: expression along restricted connection systems and synaptic localization suggest a potential role in neuronal circuitry. Dev Dyn 211:338-351.

Katz LC, Shatz CJ (1996) Synaptic activity and the construction of cortical circuits. Science 274:1133-1138.

Kelly JB, Judge PW (1994) Binaural organization of primary auditory cortex in the ferret (Mustela putorius). J Neurophysiol 71:904-913.

Kelly JB, Judge PW, Phillips DP (1986) Representation of the cochlea in primary auditory cortex of the ferret (Mustela putorius). Hear Res 24:111-115.

Kujala T, Alho K, Huotilainen M, Ilmoniemi RJ, Lehtokoski A, Leinonen A, Rinne T, Salonen O, Sinkkonen J, StandertskjöldNordenstam CG, Näätänen R (1997) Electrophysiological evidence for cross-modal plasticity in humans with early- and late-onset blindness. Psychophysiology 34:213-216.

Lessard N, Pare M, Lassonde M (1998) Early-blind human subjects localize sound sources better than sighted subjects. Nature 395:278.

Levitt P (1984) A monoclonal antibody to limbic system neurons. Science 223:229-301.

Levitt P, Barbe MF, Eagleson KL (1997) Patterning and specification of the cerebral cortex. Annu Rev Neurosci 20:1-24.

Löwel S, Singer W (1992) Selection of intrinsic horizontal connections in the visual cortex by correlated neuronal activity. Science 209-212.

Malach R, Amir Y, Harel M, Grinvald A (1993) Relationship between intrinsic connections and functional architecture revealed by optical imaging and in vivo targeted biocytin injections in primate striate cortex. Proc Natl Acad Sci USA 90:10469-10473.

Mann F, Zhukareva V, Pimenta A, Levitt P, Bolz J (1998) Membraneassociated molecules guide limbic and nonlimbic thalamocortical projections. J Neurosci 18:9409-9419.

Matsubara JA, Phillips DP (1988) Intracortical connections and their physiological correlates in the primary auditory cortex (AI) of the cat. J Comp Neurol 268:38-48.

Matsubara JA, Cynader M, Swindale NV, Stryker MP (1985) Intrinsic projections within visual cortex: Evidence for orientation-specific local connections. Proc Natl Acad Sci USA 82:935-939.

Matsubara JA, Cynader MS, Swindale NV (1987) Anatomical properties and physiological correlates of the intrinsic connections in cat area 18. J Neurosci 7:1428-1446.

Neville HJ, Schmidt A, Kutas M (1983) Altered visual-evoked potentials in congenitally deaf adults. Brain Res 266:127-132.

O'Leary DDM (1989) Do cortical areas emerge from a protocortex? Trends Neurosci 12:400-406.

Ojima H, Honda C, Jones EG (1991) Patterns of axon collateralization of identified supragranular pyramidal neurons in the cat auditory cortex. Cereb Cortex 1:80-94.

Pallas SL (1990) Cross-modal plasticity in sensory cortex: visual responses in primary auditory cortex in ferrets with induced retinal projections to the medial geniculate nucleus. In: The neocortex: ontogeny and phylogeny. NATO Advanced Research Workshop (Finlay BL, Innocenti G, Scheich H, eds), pp 205-218. New York: Plenum.

Pallas SL, Gao W-J (1999) Cross-modal reorganization of cortical connectivity. Invest Ophthalmol Vis Sci 40:S645.

Pallas SL, Moore DR (1997) Retinal axons arborize in the medial geniculate nucleus of neonatally-deafened ferrets. Soc Neurosci Abstr 23:1994.

Pallas SL, Sur M (1993) Visual projections induced into the auditory pathway of ferrets. II. Corticocortical connections of primary auditory cortex with visual input. J Comp Neurol 337:317-333.

Pallas SL, Roe AW, Sur M (1990) Visual projections induced into the auditory pathway of ferrets. I. Novel inputs to primary auditory cortex (AI) from the LP/Pulvinar complex and the topography of the MGN-AI projection. J Comp Neurol 298:50-68.

Pallas SL, Littman T, Moore DR (1999) Cross-modal reorganization of callosal connectivity in auditory cortex without altering thalamocortical input. Proc Natl Acad Sci USA 96:8751-8756.

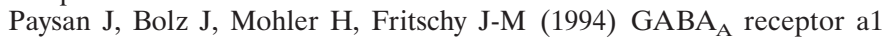
subunit, an early marker for area specification in developing rat cerebral cortex. J Comp Neurol 350:133-149.

Phillips DP, Judge PW, Kelly JB (1988) Primary auditory cortex in the ferret (Mustela putorius): neural response properties and topographic organization. Brain Res 443:281-294.

Rakic P (1988) Specification of cerebral cortical areas. Science 241:170-176.

Rauschecker JP (1995) Compensatory plasticity and sensory substitution in the cerebral cortex. Trends Neurosci 18:36-43.

Reale RA, Brugge JF, Feng JZ (1983) Geometry and orientation of neuronal processes in cat primary auditory cortex (AI) related to characteristic-frequency maps. Proc Natl Acad Sci USA 80:5449-5453.

Rebillard G, Carlier E, Rebillard M, Pujol R (1977) Enchancement of visual responses in the primary auditory cortex of the cat after an early destruction of cochlear receptors. Brain Res 129:162-164.

Rockland KS (1998) Complex microstructures of sensory cortical connections. Curr Opin Neurobiol 8:545-551.

Rockland KS, Lund JS (1982) Widespread intrinsic connections in the tree shrew visual cortex. Science 215:1532-1534.

Roe AW, Pallas SL, Hahm J, Sur M (1990) A map of visual space induced in primary auditory cortex. Science 250:818-820. 
Roe AW, Pallas SL, Kwon Y, Sur M (1992) Visual projections routed to the auditory pathway in ferrets: receptive fields of visual neurons in primary auditory cortex. J Neurosci 12:3651-3664.

Ruthazer ES, Stryker M (1996) The role of activity in the development of long-range horizontal connections in area 17 of the ferret. J Neurosci 16:7253-7269.

Sadato N, Pascual-Leone A, Grafman J, Ibañez V, Deiber MP, Dold G, Hallett M (1996) Activation of the primary visual cortex by Braille reading in blind subjects. Nature 380:526-528.

Sharma J, Angelucci A, Rao SC, Sheth BR, Sur M (1996) Auditory cortex with induced visual projections: horizontal connectivity and optical imaging of functional responses. Soc Neurosci Abstr 22:1730.

Sur M, Garraghty PE, Roe AW (1988) Experimentally induced visual projections into auditory thalamus and cortex. Science 242:1437-1441.

Sur M, Pallas SL, Roe AW (1990) Cross-modal plasticity in cortical development: differentiation and specification of sensory neocortex. Trends Neurosci 13:227-233.
Suzuki SC, Inoue T, Kimura Y, Tanaka T, Takeichi M (1997) Neuronal circuits are subdivided by differential expression of Type-II classic cadherins in postnatal mouse brains. Mol Cell Neurosci 9:433-447.

Tago H, McGeer P, McGeer E, Akiyama H, Hersh L (1989) Distribution of choline acetyltransferase immunopositive structures in the rat brainstem. Brain Res 495:271-297.

T'so DY, Gilbert CD, Wiesel TN (1986) Relationships between horizontal interactions and functional architecture in cat striate cortex as revealed by cross-correlation analysis. J Neurosci 6:1160-1170.

Wallace MN, Bajwa S (1991) Patchy intrinsic connections of the ferret primary auditory cortex. NeuroReport 2:417-420.

Wallace MN, Kitzes LM, Jones EG (1991) Intrinsic inter- and intralaminar connections and their relationship to the tonotopic map in cat primary auditory cortex. Exp Brain Res 86:527-544.

Weliky M, Katz LC (1994) Functional mapping of horizontal connections in developing ferret visual cortex: experiments and modeling. J Neurosci 14:7291-7305. 